\title{
Insight into the skew-scattering mechanism of the spin Hall effect: potential scattering versus spin-orbit scattering
}

\author{
Christian Herschbach $*$ Dmitry V. Fedorov, and Ingrid Mertig \\ Max Planck Institute of Microstructure Physics, Weinberg 2, 06120 Halle, Germany and \\ Institute of Physics, Martin Luther University Halle-Wittenberg, 06099 Halle, Germany \\ Martin Gradhand \\ H. H. Wills Physics Laboratory, University of Bristol, Bristol BS8 1TL, United Kingdom \\ Kristina Chadova, Hubert Ebert, and Diemo Ködderitzsch \\ Department of Chemistry, Physical Chemistry, Ludwig-Maximilians University Munich, Germany
}

(Dated: August 20, 2013)

\begin{abstract}
We present a detailed analysis of the skew-scattering contribution to the spin Hall conductivity using an extended version of the resonant scattering model of Fert and Levy [Phys. Rev. Lett. 106, 157208 (2011)]. For $5 d$ impurities in a $\mathrm{Cu}$ host, the proposed phase shift model reproduces the corresponding first-principles calculations. Crucial for that agreement is the consideration of two scattering channels related to $p$ and $d$ impurity states, since the discussed mechanism is governed by a subtle interplay between the spin-orbit and potential scattering in both angular-momentum channels. It is shown that the potential scattering strength plays a decisive role for the magnitude of the spin Hall conductivity.

PACS numbers: 71.15.Rf,72.25.Ba,75.76.+j,85.75.-d
\end{abstract}

An intriguing direction in the development of spintronic devices is based on the spin Hall effect (SHE) $\frac{1}{1}$ This phenomenon, caused by spin-orbit coupling (SOC), provides an opportunity for spin current generation in nonmagnetic materials without injection from ferromagnets. Materials with a large spin Hall angle (SHA), describing the efficiency of charge into spin current conversion, are highly desirable. Recently, a number of materials with a giant SHE, corresponding to SHA's of the order of 0.1 , were predicted 2.3 and observed experimentally ${ }^{4-6}$ Some of these studies ${ }^{3.6 .7}$ indicate that tuning the skew-scattering mechanism by an appropriate choice of impurities, especially in noble metals, is a promising route to obtain a giant SHE.

For the understanding of the essential conditions of such a strong SHE, a microscopic analysis of this mechanism is desired. In particular, models with input parameters provided by first-principles calculations are helpful for an intuitive picture and a detailed analysis of the material specific SHE. Recently, Fert and Levy $\stackrel{8}{\underline{8}}$ have proposed a resonant scattering model and applied it to a $\mathrm{Cu}$ host with $5 d$ impurities. The well-known behavior of the residual resistivity of noble metals with transition metal impurities is qualitatively well described by Friedel's $d$ resonance model $\underline{\underline{9}} \underline{\underline{-12}}$ Therefore, it was assumed that the spin-orbit driven transverse transport in these systems is mainly caused by impurity $d$ states $\stackrel{8}{-}$ In this paper we show that despite such a seemingly sensible assumption, the contribution related to the spin-orbit scattering in the $p$ channel is comparably large for the considered systems. Consequently, the total spin Hall conductivity (SHC) is predominantly caused by two scattering channels related to both $p$ and $d$ states. This somewhat surprising result is caused by the vertex corrections, which play an ulti- mate role for the skew-scattering mechanism. They enter the semiclassical approach via the so-called scattering-in term of the Boltzmann equation ${ }^{3,13,14}$ Furthermore, we show that for the considered $5 d$ series of impurity atoms in copper the magnitude of the SHC is mainly determined by the potential scattering strength reflected in the momentum relaxation time.

The paper is organized in three parts. First, we provide a derivation of the proposed phase shift model as an extended version of the resonant scattering model considered in Ref. 8 for a description of the SHE. Then, we present the results for $5 d$ impurities in a $\mathrm{Cu}$ host obtained within this model in comparison to ab initio results, to confirm its validity. Finally, we perform a detailed analysis identifying the significant contributions to the SHC to elucidate our findings outlined above.

Within the semiclassical approach the conductivity tensor can be written, using the spherical band approximation, as 15

$$
\hat{\sigma}=\frac{e^{2}}{V} \sum_{\mathbf{k}} \delta\left(\mathcal{E}_{\mathbf{k}}-\mathcal{E}_{F}\right) \mathbf{v}_{\mathbf{k}} \circ \boldsymbol{\Lambda}_{\mathbf{k}}=\frac{e^{2} m_{e} k_{F}}{\hbar^{2}(2 \pi)^{3}} \int \mathrm{d} \Omega_{\mathbf{k}} \mathbf{v}_{\mathbf{k}} \circ \boldsymbol{\Lambda}_{\mathbf{k}}
$$

where the mean free path is given by the Boltzmann equation 16

$$
\boldsymbol{\Lambda}_{\mathbf{k}}=\boldsymbol{\Lambda}_{\mathbf{k}}^{\text {out }}+\boldsymbol{\Lambda}_{\mathbf{k}}^{\text {in }}=\tau_{\mathbf{k}}\left(\mathbf{v}_{\mathbf{k}}+\sum_{\mathbf{k}^{\prime}} P_{\mathbf{k} \leftarrow \mathbf{k}^{\prime}} \boldsymbol{\Lambda}_{\mathbf{k}^{\prime}}\right)
$$

with the momentum relaxation time

$$
\frac{1}{\tau_{\mathbf{k}}}=\sum_{\mathbf{k}^{\prime}} P_{\mathbf{k}^{\prime} \leftarrow \mathbf{k}}=\frac{2 \pi}{\hbar} c_{i} N \sum_{\mathbf{k}^{\prime}}\left|T_{\mathbf{k}^{\prime} \leftarrow \mathbf{k}}\right|^{2} \delta\left(\mathcal{E}_{\mathbf{k}}-\mathcal{E}_{\mathbf{k}^{\prime}}\right)
$$

and the group velocity $\mathbf{v}_{\mathbf{k}}=\hbar \mathbf{k} / m_{e}$. Here, the microscopic transition probability $P_{\mathbf{k}^{\prime} \leftarrow \mathbf{k}}$ is the scattering rate 
from an initial state $\mathbf{k}$ into a final state $\mathbf{k}^{\prime}$. This quantity is defined by the corresponding transition matrix $T_{\mathbf{k}^{\prime} \leftarrow \mathbf{k}}$ and scales with the impurity concentration $c_{i}=N_{i} / N$, as valid for noninteracting impurities, $\underline{16}$

Of crucial importance for the further discussion of the considered phenomenon is the second term on the r.h.s. of Eq. (2). This scattering-in term, which corresponds to the vertex corrections of the Kubo theory in the dilute

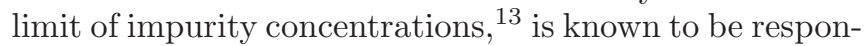
sible for the skew-scattering mechanism. $3,8,14,17$ Moreover, only the antisymmetric part of $P_{\mathbf{k} \leftarrow \mathbf{k}^{\prime}}$ involved in Eq. (2) contributes to the effect: $\frac{8,15}{}$

Following Refs. 8 and 18, the transition matrix for the "spin-up" ("+") states can be written, to first order in $\lambda_{l} / \Delta_{l}$, as

$$
\begin{array}{r}
T_{\mathbf{k}^{\prime} \leftarrow \mathbf{k}}^{+\leftarrow+}=\frac{4 \pi^{2} \hbar^{2}}{m_{e} k_{\mathrm{F}} V} \sum_{l m}\left[m \frac{\lambda_{l}}{\Delta_{l}} e^{\mathrm{i} 2 \eta_{l}} \sin ^{2} \eta_{l}-2 e^{\mathrm{i} \eta_{l}} \sin \eta_{l}\right] \\
\times\left(Y_{l}^{m}(\hat{\mathbf{k}})\right)^{*} Y_{l}^{m}\left(\hat{\mathbf{k}^{\prime}}\right) .
\end{array}
$$

Here, $\Delta_{l}$ and $\lambda_{l}$ are the resonance width and the SOC constant for the corresponding $l>0$ impurity level, respectively. This expression can be derived starting from the conventional nonrelativistic transition matrix and assuming the phase shifts to become $m$-dependent as $\eta_{l} \rightarrow \eta_{l}^{m} \approx \eta_{l}-\frac{m \lambda_{l}}{2 \Delta_{l}} \sin ^{2} \eta_{l}$ for a perturbative treatment of the SOC 18,19 Thus, in Eq. (4) the spin-orbit scattering strength is measured by $\left(\lambda_{l} / \Delta_{l}\right) \sin ^{2} \eta_{l}$, while $\sin \eta_{l}$ in the second term relates to the strength of the potential scattering. ${ }^{20}$

As mentioned above, the skew-scattering mechanism is solely determined by the antisymmetric part of the microscopic transition probability. Thus, one needs to calculate $\left|T_{\mathbf{k}^{\prime} \leftarrow \mathbf{k}}\right|_{\text {antisym }}^{2}=\left(\left|T_{\mathbf{k}^{\prime} \leftarrow \mathbf{k}}\right|^{2}-\left|T_{\mathbf{k} \leftarrow \mathbf{k}^{\prime}}\right|^{2}\right) / 2$. Since the first term on the r.h.s. of Eq. (4) is antisymmetric while the second one is symmetric with respect to exchange of $\mathbf{k}$ and $\mathbf{k}^{\prime}, \underline{\underline{8}}$ we obtain

$$
\begin{aligned}
& \left|T_{\mathbf{k}^{\prime} \leftarrow \mathbf{k}}^{+\leftarrow}\right|_{\text {antisym }}^{2}=\frac{64 \pi^{4} \hbar^{4}}{V^{2} m_{e}^{2} k_{\mathrm{F}}^{2}} \mathrm{i} \sum_{l m} \sum_{l^{\prime} m^{\prime}} m \frac{\lambda_{l}}{\Delta_{l}} \sin \left(2 \eta_{l}-\eta_{l^{\prime}}\right) \\
& \times \sin ^{2} \eta_{l} \sin \eta_{l^{\prime}} Y_{l}^{m}(\hat{\mathbf{k}})\left(Y_{l}^{m}\left(\hat{\mathbf{k}}^{\prime}\right)\right)^{*}\left(Y_{l^{\prime}}^{m^{\prime}}(\hat{\mathbf{k}})\right)^{*} Y_{l^{\prime}}^{m^{\prime}}\left(\hat{\mathbf{k}^{\prime}}\right) .
\end{aligned}
$$

Within the spherical band approximation, the skewscattering contribution to the spin Hall conductivity, $\sigma_{y x}^{s}=2 \sigma_{y x}^{+}$, is given by $\underline{15}$

$$
\sigma_{y x}^{+}=\frac{c_{i} N V e^{2} k_{\mathrm{F}}^{2} \tau_{0}^{2}}{\hbar^{3}(2 \pi)^{5}} \int \mathrm{d} \Omega_{\mathbf{k}} \int \mathrm{d} \Omega_{\mathbf{k}^{\prime}} k_{y} k_{x}^{\prime}\left|T_{\mathbf{k} \leftarrow \mathbf{k}^{\prime}}^{++}\right|_{\text {antisym }}^{2} .
$$

This is obtained assuming $\boldsymbol{\Lambda}_{\mathbf{k}^{\prime}} \approx \boldsymbol{\Lambda}_{\mathbf{k}^{\prime}}^{\text {out }}=\tau_{\mathbf{k}^{\prime}} \mathbf{v}_{\mathbf{k}^{\prime}}$ for the scattering-in term of Eq. (2) as well as an isotropic momentum relaxation time $\tau_{\mathbf{k}} \approx \tau_{0}$. The involved components of the crystal momentum can be expressed in terms of spherical harmonics as 21

$$
k_{x}=\frac{k_{\mathrm{F}} \sqrt{2 \pi}\left[Y_{1}^{-1}(\hat{\mathbf{k}})-Y_{1}^{1}(\hat{\mathbf{k}})\right]}{\sqrt{3}}, k_{y}=\frac{i k_{\mathrm{F}} \sqrt{2 \pi}\left[Y_{1}^{-1}(\hat{\mathbf{k}})+Y_{1}^{1}(\hat{\mathbf{k}})\right]}{\sqrt{3}} .
$$

Taking into account Eq. (5) and the integrals over three spherical harmonics 22 , which are related to the ClebschGordan coefficients $\stackrel{21}{\stackrel{2}{1}}$ we obtain $\underline{23}$

$$
\begin{aligned}
& \sigma_{y x}^{+}=\left(\frac{e^{2}}{\hbar}\right)\left(\frac{2 \hbar^{2} k_{\digamma}^{2} c_{i}}{\pi m_{e}^{2} V_{0}}\right) \tau_{0}^{2} \times \\
& \left\{\frac{1}{3} \frac{\lambda_{1} \sin ^{2} \eta_{1}}{\Delta_{1}}\left[\sin \left(2 \eta_{1}-\eta_{0}\right) \sin \eta_{0}-\sin \left(2 \eta_{1}-\eta_{2}\right) \sin \eta_{2}\right]\right. \\
& +\frac{\lambda_{2} \sin ^{2} \eta_{2}}{\Delta_{2}}\left[\sin \left(2 \eta_{2}-\eta_{1}\right) \sin \eta_{1}-\sin \left(2 \eta_{2}-\eta_{3}\right) \sin \eta_{3}\right] \\
& \left.+2 \frac{\lambda_{3} \sin ^{2} \eta_{3}}{\Delta_{3}} \sin \left(2 \eta_{3}-\eta_{2}\right) \sin \eta_{2}\right\},
\end{aligned}
$$

where $V_{0}$ is the unit cell volume. Here, all scattering contributions involving $s, p, d$, and $f$ states are considered, neglecting terms with $l>3$. A more general expression for $\sigma_{y x}^{+}$is provided in the Supplemental Material. $\stackrel{23}{ }$ In addition, for the isotropic momentum relaxation time involved in Eq. (8) we neglect the influence of the SOC and apply the expression 12

$$
\frac{1}{\tau_{0}}=\frac{4 \pi \hbar c_{i}}{m_{e} k_{\mathrm{F}} V_{0}} \sum_{l}(2 l+1) \sin ^{2} \eta_{l},
$$

which is similar to the approach used in Ref. 8 .

For practical applications of Eq. (8) one needs to know the SOC constant for an impurity atom in the considered host. Often this is approximated by the corresponding atomic SOC constant 8.24 However, $\lambda_{l}$ for an impurity embedded in a crystal can differ significantly from the value of an isolated atom $, 25,26$ Therefore, it is preferable to calculate $\lambda_{l}$ via the self-consistent electronic structure of a real impurity system. ${ }^{26}$ The resonance width required additionally can be obtained from the partial local density of states at the impurity site. Of course, the assumption of a simple Lorentz shape for all impurity states with $l>0$, as used in Eq. (4), is commonly difficult $\underline{10}$ However, we can generalize Eq. (8) using the following relations 27

$$
\frac{\lambda_{l} \sin ^{2} \eta_{l}}{\Delta_{l}}=\frac{2\left(\delta_{l-1 / 2}-\delta_{l+1 / 2}\right)}{2 l+1}, \quad \eta_{l}=\frac{l \delta_{l-1 / 2}+(l+1) \delta_{l+1 / 2}}{2 l+1}
$$

based on the phase shifts related to the relativistic quantum number $j=l \pm 1 / 2$. This makes it possible to apply Eq. (8) for any impurity system, since no special assumptions for the impurity states are made. Nevertheless, the virtual bound states are treated by this generalized approach as well.

For our study, the corresponding relativistic phase shifts $\delta_{l \pm 1 / 2}$ are obtained by first-principles calculations performed with a relativistic Korringa-Kohn-Rostoker Green's function method ${ }^{28}$ Their values for the considered $5 d$ impurities in a $\mathrm{Cu}$ host are presented in the Supplemental Material. 23

Figure 1 shows results for the SHC obtained using Eqs. (8)-(10) in comparison to direct first-principles calculations performed applying the Boltzmann equation ${ }^{3}$ and the Kubo-Středa formula $\underline{14}^{14}$ Also included are the corresponding results of the relativistic phase shift model, which was presented in Ref. 15. Throughout the paper, all values of the SHC are shown for the impurity concen- 


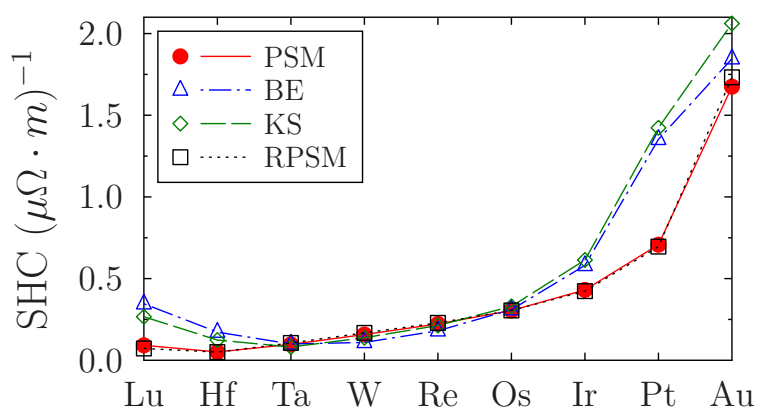

FIG. 1: (Color online) The spin Hall conductivity for $5 d$ impurities in a $\mathrm{Cu}$ host obtained by the considered phase shift model (PSM), the Boltzmann equation (BE), and the KuboStředa formula (KS). For comparison, the results obtained within the relativistic phase shift model (RPSM) are shown.

tration of 1 at.\%. The results of the considered and relativistic phase shift model almost coincide, which points to an efficient treatment of the SOC within the used perturbative approach. In addition, the models are in good agreement with the results of the ab initio calculations. This is a consequence of the almost spherical Fermi surface of copper.

In comparison to the first-principles calculations, the models give easy access to a detailed analysis which helps to identify the most important contributions. The presented model is particularly useful in that respect, since Eq. (8) makes it possible to separate different $l$ channels for the spin-orbit scattering contributing to the SHC. They are shown in Fig. 2 and labeled with $l l^{\prime}$ according to the related terms

$$
C_{l l^{\prime}}=\frac{\lambda_{l} \sin ^{2} \eta_{l}}{\Delta_{l}} \sin \left(2 \eta_{l}-\eta_{l^{\prime}}\right) \sin \eta_{l^{\prime}}
$$

in the braces of Eq. (8). Obviously, all contributions involving $s$ and $f$ states are negligible and the total SHC is provided just by two terms labeled as $p-d$ and $d-p$. Surprisingly, the $p-d$ contribution, related to the spin-orbit scattering in the $p$ channel, is of the same order as the $d-p$ contribution. Moreover, for $\mathrm{Pt}$ and $\mathrm{Au}$ impurities the $p-d$ contribution becomes even significantly larger than the $d-p$ one.

To get a clear insight into the origin of this unexpected result, it is worth to use another helpful property of Eq. (8). The Hall conductivity given by this expression is a result of the interplay between the spin-orbit and potential scattering, which were entering additively into the transition matrix only. Nevertheless, the specific structure of Eq. (8) still allows one to separate out the spin-orbit scattering strength from the rest of the skewscattering contribution to the SHC. In other words, the terms $C_{p-d} \equiv-\frac{1}{3} C_{12}$ and $C_{d-p} \equiv C_{21}$ providing the dominant contributions to Eq. (8) can be divided into two factors. The first one, $\left(\lambda_{l} / \Delta_{l}\right) \sin ^{2} \eta_{l}$, is the spinorbit scattering strength for the corresponding $l$ channel, while the rest is described merely in terms of the nonrelativistic phase shifts. Figure 3 shows the two discussed

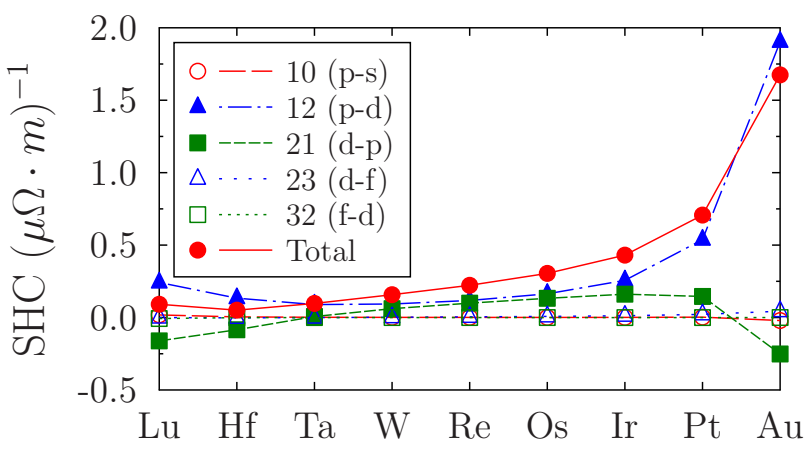

FIG. 2: (Color online) The spin Hall conductivity for $5 d$ impurities in a $\mathrm{Cu}$ host obtained by the considered phase shift model. The different contributions to $\sigma_{y x}^{s}$ labeled as $l l^{\prime}$ correspond to $C_{l l^{\prime}}$ defined by Eq. (11).

parts separately. In contrast to the SOC part related to $l=1$, which has just a small enhancement going from $\mathrm{Lu}$ to $\mathrm{Au}$, the corresponding part for $l=2$ shows the resonance behavior ${ }^{9,10}$ caused by the $5 d$ shell filling (see Table S2 in the Supplemental Material ${ }^{23}$ ). This part is enhanced around W and reduces towards both sides. As a result, in the case of $\mathrm{Au}$ impurities the spin-orbit scattering strength in the $p$ channel is four times larger than for $d$ states. However, from Hf to Ir both contributions are of comparable magnitude and how they add up is determined by the second part of the corresponding $C_{l l^{\prime}}$ terms, which is given by

$$
\begin{aligned}
& \sin \left(2 \eta_{l}-\eta_{l^{\prime}}\right) \sin \eta_{l^{\prime}} \\
& \quad=2 \sin \eta_{l} \sin \eta_{l^{\prime}} \cos \left(\eta_{l}-\eta_{l^{\prime}}\right)-\sin ^{2} \eta_{l^{\prime}} .
\end{aligned}
$$

Here, the used identity helps us to highlight a further important point, since the first term on the r.h.s of Eq. (12) is the same for $l l^{\prime}$ and $l^{\prime} l$ contributions. The difference is caused solely by the second term, which depends on $\eta_{2}$ and $\eta_{1}$ for the $p-d$ and $d-p$ contributons, respectively. Thus, the nature of the scattering-in term, which is responsible for the structure of Eq. (6), leads to the coupling of the spin-orbit scattering in the $p$ channel with the potential scattering in the $d$ channel. For the considered systems, the latter one is enhanced by the resonance properties of the $5 d$ impurities in copper. In contrast to the results of Ref. 8, the subtle combination of the two scattering mechanisms causes a positive SHC, and consequently a positive SHA, for all the considered systems.

Moreover, the main trend of the total SHC with a significant enhancement for $\mathrm{Pt}$ and $\mathrm{Au}$, as seen in Figs. 1 and 2, is entirely provided by the behavior of $\tau_{0}$. This is illustrated by Fig. 4 where we show the momentum relaxation time in addition to the two dominant contributions in the braces of Eq. (8). With this expression, the strong correlation between $\tau_{0}^{2}$ and the total SHC of Figs. 1 and 2 points to the crucial role of the potential scattering strength by itself for the magnitude of the SHC.

In summary, we developed a phase shift model for the skew-scattering mechanism of the SHE as an extension of 


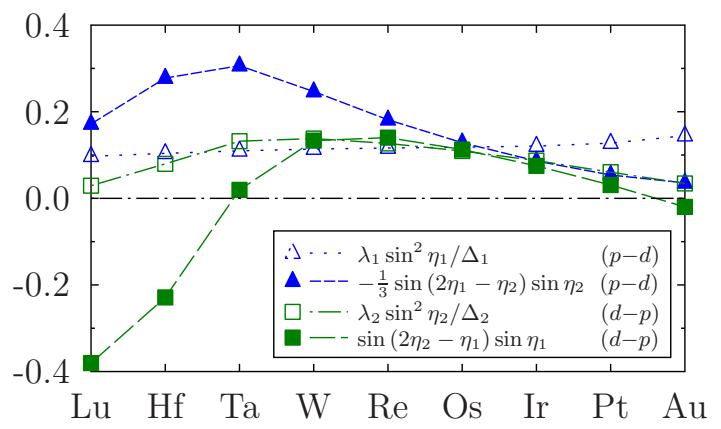

FIG. 3: (Color online) The two parts of the contributions $C_{p-d}$ and $C_{d-p}$ are shown separately.

the resonant scattering model of Ref. 8. Exploiting this approach we show that the spin Hall conductivity results from a subtle interplay between the spin-orbit and potential scattering in different $l$ channels, caused by the structure of the scattering-in term (vertex corrections). For $5 d$ impurities in copper this leads to a crucial importance of the contribution related to the spin-orbit coupling of $p$ states, which explains the unanticipated behavior of the spin Hall conductivity. The proposed phase shift model provides good agreement with results of first-principles approaches and highlights the complexity of the specific problem. In combination with parameters derived from $a b$ initio calculations this model can be used for a simplified description of the SHE in dilute alloys based on noble metals. However, we emphasize the utmost importance to carefully consider all leading terms.

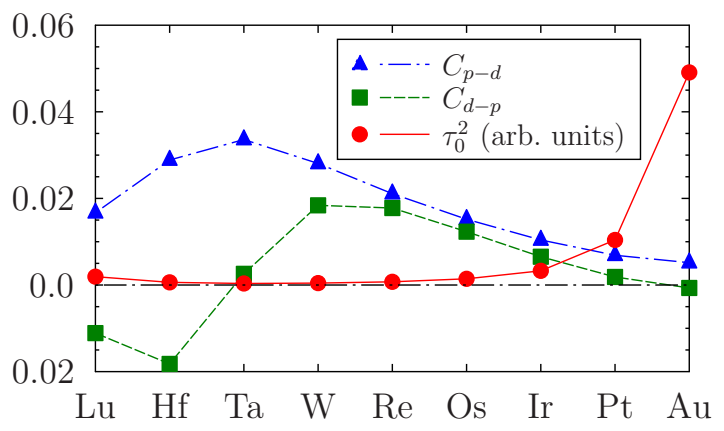

FIG. 4: (Color online) The contributions $C_{p-d}$ and $C_{d-p}$ are shown versus $\tau_{0}^{2}$ (in arbitrary units).

The work was partially supported by the Deutsche Forschungsgemeinschaft (DFG) via SFB 762. The authors K.C., H.E. and D.K. acknowledge support from the DFG via SFB 689 and SPP 1538. In addition, M.G. acknowledges financial support from the DFG via a research fellowship (GR3838/1-1).
* Electronic address: cherschb@mpi-halle.mpg.de

1 M. I. Dyakonov and V. Perel, Phys. Lett. A 35, 459 (1971); J. E. Hirsch, Phys. Rev. Lett. 83, 1834 (1999).

2 T. Tanaka, H. Kontani, M. Naito, T. Naito, D. S. Hirashima, K. Yamada and J. Inoue, Phys. Rev. B 77, 165117 (2008)

3 M. Gradhand, D. V. Fedorov, P. Zahn, and I. Mertig, Phys. Rev. Lett. 104, 186403 (2010); Phys. Rev. B 81, 245109 (2010).

4 T. Seki, Y. Hasegawa, S. Mitani, S. Takahashi, H. Imamura, S. Maekawa, J. Nitta, and K. Takanashi, Nature Mater. 7, 125 (2008).

${ }^{5}$ L. Liu, C.-F. Pai, Y. Li, H. W. Tseng, D. C. Ralph, and R. A. Buhrman, Science 336, 555 (2012).

${ }^{6}$ Y. Niimi, Y. Kawanishi, D. H. Wei, C. Deranlot, H. X. Yang, M. Chshiev, T. Valet, A. Fert and Y. Otani, Phys. Rev. Lett. 109, 156602 (2012).

7 M. Gradhand, D. V. Fedorov, P. Zahn, I. Mertig, Y. Otani, Y. Niimi, L. Vila, and A. Fert, SPIN 2, 1250010 (2012).

8 A. Fert and P. M. Levy, Phys. Rev. Lett. 106, 157208 (2011)

9 J. Friedel, Nuovo Cimento Suppl. 7 , 287 (1958).

10 E. Daniel and J. Friedel, in Proc. Intern. Conf. On Low Temperature Physics, edited by J. Daunt, P. Edwards, F. Milford, M. Yaqub (Plenum, New York, 1965), p. 933.

11 P. J. Braspenning, R. Zeller, P. H. Dederichs, and A. Lodder, J. Phys. F: Met. Phys. 12, 105 (1982).

12 I. Mertig, E. Mrosan, and P. Ziesche, Multiple Scattering Theory of Point Defects in Metals: Electronic Properties (Teubner, Leipzig, 1987).
13 W. H. Butler, Phys. Rev. B 31, 3260 (1985).

14 S. Lowitzer, M. Gradhand, D. Ködderitzsch, D. V. Fedorov, I. Mertig, and H. Ebert, Phys. Rev. Lett. 106, 056601 (2011).

15 D. V. Fedorov, C. Herschbach, A. Johansson, S. Ostanin, I. Mertig, M. Gradhand, K. Chadova, D. Ködderitzsch, and H. Ebert, accepted to Phys. Rev. B (2013).

16 I. Mertig, Rep. Prog. Phys. 62, 237 (1999).

17 N. A. Sinitsyn, J. Phys.: Cond. Matter 20, 023201 (2008).

18 A. Fert, A. Friederich, and A. Hamzic, J. Magn. Magn. Mater. 24, 231 (1981).

19 A. Fert and O. Jaoul, Phys. Rev. Lett. 28, 303 (1972).

20 For sake of simplicity, here we use the name potential scattering for both potential and resonance scattering considered within the conventional nonrelativistic treatment 10

21 D. A. Varshalovich, A. N. Moskalev, and V. K. Khersonskii, Quantum Theory of Angular Momentum (World Scientific, Singapore, 1988).

22 A. Fert and P. M. Levy, Phys. Rev. B 36, 1907 (1987).

23 See Supplemental Material at ... for details of the application of the considered phase shift model.

24 P. Monod and S. Schultz, J. Phys. (Paris) 43, 393 (1982).

${ }^{25}$ Y. Yafet, in Solid State Physics, Vol. 14, F. Seitz and D. Turnbull, Eds. (Academic Press, New York, 1963).

26 D. V. Fedorov, P. Zahn, M. Gradhand, and I. Mertig, Phys. Rev. B 77, 092406 (2008); 79, 059901(E) (2009).

27 Y. Yafet, J. Appl. Phys. 39, 853 (1968).

28 M. Gradhand, M. Czerner, D. V. Fedorov, P. Zahn, B. Y. Yavorsky, L. Szunyogh, and I. Mertig, Phys. Rev. B 80, 224413 (2009). 